\title{
Metastable States of Molecules
}

\author{
E. B. Davies \\ St. John's College, Oxford OX1 3JP, England
}

\begin{abstract}
We define metastable states to be density matrices which are at local minima of a certain non-linear functional, and investigate their general properties, proving in particular that the metastable states are not necessarily unique but are "modified Gibbs states". The case of an atom in an external electric field is investigated in some detail.
\end{abstract}

\section{Introduction}

We consider a molecule composed of $(N+1)$ spinless, distinguishable, threedimensional particles in the centre of mass coordinate system. The Hamiltonian $H$ on the Hilbert space $\mathscr{H}=L^{2}\left(\mathbb{R}^{3 N}\right)$ is

$$
H=H_{0}+V\left(x_{1}, \ldots, x_{N}\right),
$$

where the internal kinetic energy operator $H_{0}$ of the molecule is translation invariant and satisfies

$$
-\delta \Delta \leqq H_{0} \leqq-\delta^{-1} \Delta
$$

for some $\delta>0$, and we assume that the potential $V$ arises from pair interactions between the various particles, and has form bound zero with respect to $-\Delta$, so that (1.1), interpreted as a form sum, defines $H$ as a semibounded self-adjoint operator.

If the set of mixed states $\varrho$ on $\mathscr{H}$ is defined by

$$
X=\{\varrho: \varrho \geqq 0 \text { and } \operatorname{tr}[\varrho]=1\}
$$

then the entropy of a state $\varrho$ is

$$
S(\varrho)=-\operatorname{tr}[\varrho \log \varrho]
$$

and its free energy at the inverse temperature $\beta>0$ is

$$
\operatorname{tr}[H \varrho]-\beta^{-1} S(\varrho) \text {. }
$$


Formally speaking the free energy has a unique minimum on $X$, at the Gibbs state

$$
\varrho=e^{-\beta H} / \operatorname{tr}\left[e^{-\beta H}\right] .
$$

However for real molecules this is not actually the case because the trace is not finite, so the minimising state does not exist. This is because even at very low temperatures the possibility of dissociation is non-zero, so this process must eventually occur, and no stable state exists.

The same problem occurs even at zero temperature for a molecule in a weak external electric field, because the Hamiltonian is not bounded below and possesses no ground state. However, there may still exist metastable states, or resonances, which remain effectively bound for very long periods of time. While it is possible to define resonances in terms of poles of an analytic continuation of the Hamiltonian [3--5], the relationship of this approach to more operational definitions is not clear. One possible physical criterion for a metastable state is that it is a square-integrable wave function whose energy spread is very narrow, so that it is persistent in time, and whose distribution in position space is very concentrated [6].

Similarly the precise definition of metastable states in classical statistical mechanics is by no means obvious, although several criteria for their recognition are available $[8,11]$. One approach is to minimise the free energy for states restricted to an appropriate region of configuration space, which is specified by disallowing clusters above a certain critical size [1]. All current approaches seem to depend upon imposing constraints on the allowed states, and therefore involve a degree of arbitrariness (or physical insight) in the choice of those constraints. Our present approach is no exception.

Following an idea in [2], we define the variance in configuration space of a state $\varrho \in X$ to be

$$
W(\varrho)=\sum_{r=1}^{3 N}\left\{\operatorname{tr}\left[Q_{r}^{2} \varrho\right]-\operatorname{tr}\left[Q_{r} \varrho\right]^{2}\right\},
$$

where $Q_{r}$ are the position operators on $\mathscr{H}$. The non-linear functional $W$ on $X$ is both translationally and rotationally invariant. We then seek to minimise the functional

$$
\mathscr{E}(\varrho)=\operatorname{tr}[H \varrho]-\beta^{-1} S(\varrho)+\alpha W(\varrho),
$$

where $0<\alpha, \beta<\infty$. The parameter $\alpha$ is called the inverse stability of a local minimum $\varrho \in X$ and $\beta$ is called its inverse temperature. If we put $\alpha=0$ we obtain the Gibbs state as the unique minimum if the trace is finite, and say that it is infinitely stable. If we put $\beta^{-1}=0$ then we reduce to the analysis of what were called "stable ground states" in [2]. In this paper we consider the case $0<\alpha, \beta<\infty$, so that the functional $\mathscr{E}$ on $X$ is neither convex nor concave. One of our major goals will be to determine when $\mathscr{E}$ has local minima in $X$ and to investigate the stability, or persistence, of these minima under the linear time evolution defined by $H$. We do not consider here the non-linear Schrodinger equation associated with $\mathscr{E}$ in [2].

While our procedure is to some extent ad hoc, it has the merit of identifying a class of states depending upon only two thermodynamic parameters, $\alpha$ and $\beta$. 
Moreover, we shall see that the explicit construction of these states is quite feasible, and that they may be regarded as "modified Gibbs states".

Another obvious method of circumventing the fact, mentioned above, that the trace of $e^{-\beta H}$ is infinite, is to put the whole system in a box. While this may be numerically more accurate (in some sense), it is slightly more complicated in that one needs to determine two parameters, the centre and diameter of the box. The main advantage of our approach, however, is that it suggests a possible method of determining all stable molecules obtainable from a given set of atoms. As was indicated in [2], even after fixing $\alpha>0$ it is possible that $\mathscr{E}$ has several different local minima, and that these might correspond to chemically distinguishable configurations of the atoms, known as structural isomers.

\section{Lower Semi-continuity of $\mathscr{E}$}

We start by recalling some well-known properties of entropy and relative entropy. Let $\left\{f_{r}\right\}$ be an orthonormal basis of eigenvectors for $\varrho \in X$ and let $\varrho f_{r}=\lambda_{r} f_{r}$, so that $\lambda_{r} \geqq 0$ and

$$
\operatorname{tr}[\varrho]=\sum_{r} \lambda_{r}=1 .
$$

Let $\left\{g_{s}\right\}$ be an orthonormal basis of eigenvectors of $\sigma \in X$ and let $\sigma g_{s}=\mu_{s} g_{s}$, so that

$$
\operatorname{tr}[\sigma]=\sum_{s} \mu_{s}=1
$$

Suppose also that $\sigma$ is strictly positive, or equivalently that $\mu_{s}>0$ for all $s$. The entropy functional $S$ on $X$ is defined by

$$
S(\varrho)=-\sum \lambda_{r} \log \lambda_{r}
$$

and satisfies

$$
0 \leqq S(\varrho) \leqq+\infty
$$

Moreover $S$ is a concave functional on $X$ and is lower semi-continuous with respect to the trace norm topology of $X[13]$. The relative entropy functional is defined by

$$
S(\varrho \mid \sigma)=\sum_{r, s}\left|\left\langle f_{r}, g_{s}\right\rangle\right|^{2}\left\{\lambda_{r} \log \lambda_{r}-\lambda_{r} \log \mu_{s}-\lambda_{r}+\mu_{s}\right\}
$$

so that

$$
0 \leqq S(\varrho \mid \sigma) \leqq+\infty
$$

The relative entropy is jointly convex with respect to $\varrho$ and $\sigma$ and is also lower semi-continuous $[7,13]$.

If $K$ is a non-negative self-adjoint operator on $\mathscr{H}$ we define

$$
\operatorname{tr}[K \varrho]=\sum \lambda_{r}\left\|K^{1 / 2} f_{r}\right\|^{2}
$$

so that

$$
0 \leqq \operatorname{tr}[K \varrho] \leqq+\infty .
$$


Moreover

$$
\operatorname{tr}[K \varrho]=\lim _{n \rightarrow \infty} \operatorname{tr}\left[K_{n} \varrho\right],
$$

where

$$
K_{n}=K\left(1+n^{-1} K\right)^{-1}
$$

is a monotonically increasing sequence of bounded self-adjoint operators on $\mathscr{H}$. Hence $\operatorname{tr}[K \varrho]$ is a linear and lower semi-continuous function of $\varrho \in X$. that

In order to relate $S(\varrho \mid \sigma)$ to the free energy of $\varrho$ we suppose that $\sigma=e^{-K}$, and

$$
K g_{s}=k_{s} g_{s}
$$

so that

$$
\mu_{\mathrm{s}}=\exp \left(-k_{\mathrm{s}}\right) \text {. }
$$

We also suppose that

$$
\operatorname{tr}\left[e^{-\gamma K}\right]<\infty
$$

for all $0<\gamma<\infty$.

Lemma 1. $S(\varrho \mid \sigma)<\infty$ if and only if $\operatorname{tr}[K \varrho]<\infty$. If this holds then

$$
S(\varrho \mid \sigma)=\operatorname{tr}[K \varrho]-S(\varrho) \text {. }
$$

Moreover if $0<\gamma<1$ then

$$
S(\varrho \mid \sigma) \leqq \operatorname{tr}[K \varrho] \leqq(1-\gamma)^{-1}\left\{S(\varrho \mid \sigma)+\log \operatorname{tr}\left[e^{-\gamma K}\right]\right\} .
$$

Proof. Since

$$
\operatorname{tr}[\varrho]=\operatorname{tr}[\sigma]=1
$$

we have

$$
\begin{aligned}
S(\varrho \mid \sigma) & =\sum_{r, s}\left|\left\langle f_{r}, g_{s}\right\rangle\right|^{2}\left\{\lambda_{r} \log \lambda_{r}+\lambda_{r} k_{s}\right\} \\
& \leqq \sum_{r, s}\left|\left\langle f_{r}, g_{s}\right\rangle\right|^{2} \lambda_{r} k_{s} \\
& =\sum_{r} \lambda_{r}\left\|K^{1 / 2} f_{r}\right\|^{2} \\
& =\operatorname{tr}[K \varrho] .
\end{aligned}
$$

Now put

$$
\mu=e^{-\gamma \mathbf{K}} / \operatorname{tr}\left[e^{-\gamma K}\right]
$$

so that $\mu \in X$ and

$$
0 \leqq S(\varrho \mid \mu)=\sum_{r, s}\left|\left\langle f_{r}, g_{s}\right\rangle\right|^{2}\left\{\lambda_{r} \log \lambda_{r}+\lambda_{r} \gamma k_{s}+\lambda_{r} \log \operatorname{tr}\left[e^{-\gamma K}\right]\right\} .
$$


Therefore

$$
\begin{aligned}
(1-\gamma) \operatorname{tr}[K \varrho] & \leqq \sum_{r, s}\left|\left\langle f_{r}, g_{s}\right\rangle\right|^{2}\left\{\lambda_{r} \log \lambda_{r}+\lambda_{r} k_{s}+\lambda_{r} \log \operatorname{tr}\left[e^{-\gamma K}\right]\right\} \\
& =S(\varrho \mid \sigma)+\log \operatorname{tr}\left[e^{-} \gamma^{K}\right] .
\end{aligned}
$$

The lemma follows from these two inequalities.

Lemma 2. If $S(\varrho \mid \sigma)<\infty$ then $S(\varrho)<\infty$. If $\left\|\varrho_{n}-\varrho\right\|_{1} \rightarrow 0$ and $S\left(\varrho_{n} \mid \sigma\right)$ is a bounded sequence then

$$
\lim _{n \rightarrow \infty} S\left(\varrho_{n}\right)=S(\varrho)
$$

Proof. The first statement follows from the proof of the last lemma. The second statement may be found in [13, p. 241].

We now turn to the definition of the variance $W(\varrho)$. We put $W(\varrho)=+\infty$ unless $\operatorname{tr}\left[Q_{r}^{2} \varrho\right]<\infty$ for all $r$, and in that case put

$$
W(\varrho)=\sum_{r=1}^{3 N}\left\{\operatorname{tr}\left[Q_{r}^{2} \varrho\right]-\operatorname{tr}\left[Q_{r} \varrho\right]^{2}\right\}
$$

Lemma 3. The functional $W$ is concave and lower semi-continuous on $X$.

Proof. Concavity is trivial. To prove lower semi-continuity suppose that $\left\|\varrho_{n}-\varrho\right\|_{1} \rightarrow 0$ and that

$$
c=\liminf _{n \rightarrow \infty} W\left(\varrho_{n}\right) .
$$

By passing to a subsequence and eliminating trivial cases we can assume that

$$
\lim _{n \rightarrow \infty} W\left(\varrho_{n}\right)=c<\infty .
$$

If $\operatorname{tr}\left[Q_{r} Q_{n}\right]$ is unbounded for some $r$ as $n \rightarrow \infty$, then by passing to another subsequence we can assume that

$$
a_{n}=\left|\operatorname{tr}\left[Q_{r} \varrho_{n}\right]\right| \rightarrow \infty
$$

as $n \rightarrow \infty$. If $P_{R}$ is the projection associated with the region $\{x:|x| \leqq R\}$ of configuration space then

$$
\operatorname{tr}\left[P_{R} \varrho_{n}\right]\left(a_{n}-R\right)^{2} \leqq \operatorname{tr}\left[Q_{r}^{2} \varrho_{n}\right]-\operatorname{tr}\left[Q_{r} \varrho_{n}\right]^{2}
$$

for large enough $n$. Equations (2.1) and (2.2) now imply that

$$
\lim _{n \rightarrow \infty} \operatorname{tr}\left[P_{R} \varrho_{n}\right]=0 .
$$

Since $R<\infty$ is arbitrary, we deduce that $\varrho_{n}$ converges weakly to zero, which is incompatible with $\left\|\varrho_{n}-\varrho\right\|_{1} \rightarrow 0$. The contradiction implies that there exists $a<\infty$ such that

$$
\left|\operatorname{tr}\left[Q_{r} Q_{n}\right]\right| \leqq a
$$


for all $r$ and $n$. Hence

$$
\limsup _{n \rightarrow \infty} \operatorname{tr}\left[\left(\sum_{r} Q_{r}^{2}\right) \varrho_{n}\right] \leqq c+3 N a^{2} .
$$

This implies that

$$
\operatorname{tr}\left[\left(\sum_{r} Q_{r}^{2}\right) \varrho\right] \leqq \liminf _{n \rightarrow \infty} \operatorname{tr}\left[\left(\sum_{r} Q_{r}^{2}\right) \varrho_{n}\right]
$$

and that

$$
\operatorname{tr}\left[Q_{r} \varrho\right]=\lim _{n \rightarrow \infty} \operatorname{tr}\left[Q_{r} \varrho_{n}\right]
$$

for all $r$. The last two equations imply the lower semi-continuity of $W$.

We now adopt the convention

$$
Q^{2}=\sum_{r=1}^{3 N} Q_{r}^{2}
$$

the right-hand side being defined as the form sum.

Corollary 4. If $\varrho_{n} \in X$ and $\left\|\varrho_{n}-\varrho\right\|_{1} \rightarrow 0$ then $W\left(\varrho_{n}\right)$ is a bounded sequence if and only if $\operatorname{tr}\left[Q^{2} \varrho_{n}\right]$ is a bounded sequence. In this case one has

$$
\lim _{n \rightarrow \infty} \operatorname{tr}\left[Q_{r} \varrho_{n}\right]=\operatorname{tr}\left[Q_{r} \varrho\right]
$$

for all $r$.

We combine ideas from all the above lemmas in our definition of $\mathscr{E}$. We put

$$
K=\beta\left(H+\alpha Q^{2}\right)+\log \operatorname{tr}\left[e^{-\beta\left(H+\alpha Q^{2}\right)}\right]
$$

so that $\operatorname{tr}\left[e^{-K}\right]=1$, and $\operatorname{tr}\left[e^{-\gamma K}\right]<\infty$ for all $0<\gamma<\infty$. The finiteness of the traces follows from the form inequality

$$
H+\alpha Q^{2} \geqq \varepsilon\left(-\Delta+Q^{2}\right)-\varepsilon^{-1} 1
$$

derivable for some $\varepsilon>0$ from our hypotheses on $H$.

If $\varrho \in X$ and $\operatorname{tr}[K \varrho]=+\infty$ we put $\mathscr{E}(\varrho)=+\infty$. If $\operatorname{tr}[K \varrho]<\infty$ then $\operatorname{tr}\left[Q^{2} \varrho\right]<\infty$ so $\operatorname{tr}\left[Q_{r} \varrho\right]$ is well-defined and finite for all $r$. Moreover $S(\varrho)$ is finite by Lemmas 1 and 2 so we can put

$$
\begin{aligned}
\mathscr{E}(\varrho) & \left.=\operatorname{tr}\left[H+\alpha Q^{2}\right) \varrho\right]-\beta^{-1} S(\varrho)-\alpha \sum_{r} \operatorname{tr}\left[Q_{r} \varrho\right]^{2} \\
& =\operatorname{tr}[H \varrho]-\beta^{-1} S(\varrho)+\alpha W(\varrho) \\
& =\beta^{-1} S\left(\varrho \mid e^{-K}\right)-\beta^{-1} \log \operatorname{tr}\left[e^{-\beta\left(H+\alpha Q^{2}\right)}\right]-\alpha \sum_{r} \operatorname{tr}\left[Q_{r} \varrho\right]^{2} .
\end{aligned}
$$

Theorem 5. The functional

$$
\mathscr{E}: X \rightarrow(-\infty, \infty]
$$

is lower semi-continuous and bounded below. 
Proof. There exists $\delta>0$ such that $\delta H_{0} \leqq H \leqq \delta^{-1} H_{0}$. If we define $\mathscr{E}_{0}$ by replacing $H$ by $\delta H_{0}$ then it is clear that $\mathscr{E}(\varrho)<\infty$ if and only if $\mathscr{E}_{0}(\varrho)<\infty$, and that

$$
\mathscr{E}(\varrho) \geqq \mathscr{E}_{0}(\varrho)
$$

for all $\varrho \in X$. Since $\mathscr{E}_{0}$ is invariant under space translations

$$
\begin{aligned}
\inf & \left\{\mathscr{E}_{0}(\varrho): \varrho \in X\right\} \\
& =\inf \left\{\mathscr{E}_{0}(\varrho): \operatorname{tr}\left[Q_{r} \varrho\right]=0 \text { all } r\right\} \\
& \left.=\inf \left\{\operatorname{tr}\left[\delta H_{0}+\alpha Q^{2}\right) \varrho\right]-\beta^{-1} S(\varrho): \operatorname{tr}\left[Q_{r} \varrho\right]=0 \text { all } r\right\} \\
& \geqq \inf \left\{\operatorname{tr}\left[\left(\delta H_{0}+\alpha Q^{2}\right) \varrho\right]-\beta^{-1} S(\varrho): \varrho \in X\right\} \\
& =-\beta^{-1} \log \operatorname{tr}\left[e^{-\beta\left(\delta H_{0}+\alpha Q^{2}\right)}\right]
\end{aligned}
$$

the inf being achieved by a Gibbs state.

To prove lower semi-continuity of $\mathscr{E}$ we assume that $\left\|\varrho_{n}-\varrho\right\|_{1} \rightarrow 0$ and that

$$
\mathscr{E}\left(\varrho_{n}\right) \leqq c<\infty
$$

for all $n$. If $\varrho_{n}^{\prime}$ are space translates of $\varrho_{n}$ normalized so that

$$
\operatorname{tr}\left[Q_{r} Q_{n}^{\prime}\right]=0
$$

for all $r$ and $n$, then

$$
\begin{array}{r}
\operatorname{tr}\left[\left(\delta H_{0}+\alpha Q^{2}\right) \varrho_{n}^{\prime}\right]-\beta^{-1} S\left(\varrho_{n}^{\prime}\right) \\
\quad=\mathscr{E}_{0}\left(\varrho_{n}^{\prime}\right)=\mathscr{E}_{0}\left(\varrho_{n}\right) \leqq \mathscr{E}\left(\varrho_{n}\right) \leqq c
\end{array}
$$

for all $n$. Lemma 1 implies that $\operatorname{tr}\left[\left(\delta H_{0}+\alpha Q^{2}\right) \varrho_{n}^{\prime}\right]$ is a bounded sequence and that

$$
S\left(\varrho_{n}\right)=S\left(\varrho_{n}^{\prime}\right)
$$

is also a bounded sequence. Moreover

$$
W\left(\varrho_{n}\right)=W\left(\varrho_{n}^{\prime}\right)=\operatorname{tr}\left[Q^{2} \varrho_{n}^{\prime}\right]
$$

is a bounded sequence, so Corollary 4 implies that

$$
\lim _{n \rightarrow \infty} \operatorname{tr}\left[Q_{r} \varrho_{n}\right]=\operatorname{tr}\left[Q_{r} \varrho\right]
$$

for all $r$. This equation combined with (2.3) and the lower semi-continuity of $S\left(\varrho \mid e^{-K}\right)$ implies

$$
\liminf _{n \rightarrow \infty} \mathscr{E}\left(\varrho_{n}\right) \geqq \mathscr{E}(\varrho) .
$$

Corollary 6. If $\left\|\varrho_{n}-\varrho\right\|_{1} \rightarrow 0$ and $\mathscr{E}\left(\varrho_{n}\right)$ is a bounded sequence then

$$
\lim S\left(\varrho_{n}\right)=S(\varrho), \quad \lim _{n \rightarrow \infty} \operatorname{tr}\left[Q_{r} \varrho_{n}\right]=\operatorname{tr}\left[Q_{r} \varrho\right]
$$

for all $r$.

The lower semi-continuity of $\mathscr{E}$ does not imply that it has a minimum on $X$, since $X$ is not compact in the trace norm topology. However the existence of local minima may be demonstrated using the following lemma. 
Lemma 7. If $L \geqq 0$ is a self-adjoint operator such that $(L+1)^{-1 / 2}$ is compact then for every $c<\infty$ the set

$$
S=\{\varrho \in X: \operatorname{tr}[(L+1) \varrho] \leqq c\}
$$

is compact in the trace norm topology.

Proof. If $\mathscr{T}(\mathscr{H})$ is the Banach space of trace class operators then the bounded linear operator $\mathscr{L}$ on $\mathscr{T}(\mathscr{H})$ defined by

$$
\mathscr{L}(\sigma)=(L+1)^{-1 / 2} \sigma(L+1)^{-1 / 2}
$$

is compact. Therefore

$$
S=X \cap \mathscr{L}\{\sigma \geqq 0: \operatorname{tr}[\sigma] \leqq c\}
$$

has compact closure. Since $S$ is actually closed, it is compact.

Theorem 8. If $\Omega$ is a compact subset of $\mathbb{R}^{3 N}$ then $\mathscr{E}$ has a finite minimum in the set $X_{\Omega}=\{\varrho \in X: \mathscr{E}(\varrho)<\infty$ and $\operatorname{tr}[Q \varrho] \in \Omega\}$.

Proof. Suppose that $\varrho_{n} \in X_{\Omega}$ satisfy

$$
\lim _{n \rightarrow \infty} \mathscr{E}\left(\varrho_{n}\right)=\inf \left\{\mathscr{E}(\varrho): \varrho \in X_{\Omega}\right\} .
$$

Then (2.3) implies that $S\left(\varrho_{n} \mid e^{-K}\right)$ is a bounded sequence so Lemma 1 implies that $\operatorname{tr}\left[\left(H+\alpha Q^{2}\right) \varrho_{n}\right]$ is a bounded sequence. Since $\left(H+\alpha Q^{2}+\lambda\right)^{-1 / 2}$ is compact for some $\lambda>0$, Lemma 7 implies that there is a trace norm convergent subsequence of $\varrho_{n}$. The proof is now completed by using the lower semi-continuity of $\mathscr{E}$ on this sequence.

The following easy modification of the above theorem is useful for applications to molecules in an external electric field. The extra term is handled by using Corollary 6.

Theorem 9. If $\Omega$ is a compact subset of $\mathbb{R}^{3 n}$ and $A \in R^{3 N}$ then the functional

$$
\mathscr{E}^{\prime}(\varrho)=\mathscr{E}(\varrho)-\sum_{r=1}^{3 N} A_{r} \operatorname{tr}\left[Q_{r} \varrho\right]
$$

has a finite minimum in the set $X_{\Omega}$.

\section{Definition of Metastable States}

A state $\varrho_{0} \in X$ is said to be a metastable state with inverse stability $\alpha$ and inverse temperature $\beta$ if there exists $\varepsilon>0$ such that $\left\|\varrho-\varrho_{0}\right\|<\varepsilon$ implies

$$
\mathscr{E}(\varrho) \geqq \mathscr{E}\left(\varrho_{0}\right) .
$$

In order to identify the metastable states we introduce the real-valued function $E$ on $\mathbb{R}^{3 N}$, defined by

$$
\begin{aligned}
E(x) & =\min \{\mathscr{E}(\varrho): \operatorname{tr}[Q \varrho]=x\} \\
& =\min \left\{\mathscr{F}_{x}(\varrho): \operatorname{tr}[Q \varrho]=x\right\},
\end{aligned}
$$

where

$$
\mathscr{F}_{x}(\varrho)=\operatorname{tr}\left[\left\{H+\alpha(Q-x)^{2}\right\} \varrho\right]-\beta^{-1} S(\varrho) .
$$


The existence of the minimum follows from Theorem 8. Its uniqueness is a consequence of the fact that for each $x \in \mathbb{R}^{3 N}, \mathscr{F}_{x}$ is a free energy functional on $X$ and so is strictly convex. We denote the state which minimizes (3.1) by $\varrho_{x}$.

Lemma 10. For all $a \in \mathbb{R}^{3 N}$ the function

$$
E(x)+\alpha(x-a)^{2}
$$

is convex.

Proof. It is sufficient to consider the case $a=0$. If $0<\lambda<1$ and $y, z \in \mathbb{R}^{3 N}$ and

$$
x=(1-\lambda) y+\lambda z
$$

and

$$
\sigma=(1-\lambda) \varrho_{y}+\lambda \varrho_{z}
$$

then

$$
\operatorname{tr}[Q \sigma]=x .
$$

The equalities

$$
\begin{aligned}
E(x)+\alpha x^{2} & =\min \left\{\operatorname{tr}\left[\left(H+\alpha Q^{2}\right) \varrho\right]-\beta^{-1} S(\varrho): \operatorname{tr}[Q \varrho]=x\right\} \\
& =\operatorname{tr}\left[\left(H+\alpha Q^{2}\right) \varrho_{x}\right]-\beta^{-1} S\left(\varrho_{x}\right)
\end{aligned}
$$

imply that

$$
\begin{aligned}
E(x)+\alpha x^{2} \leqq & \operatorname{tr}\left[\left(H+\alpha Q^{2}\right) \sigma\right]-\beta^{-1} S(\sigma) \\
\leqq & (1-\lambda)\left\{\operatorname{tr}\left[\left(H+\alpha Q^{2}\right) \varrho_{y}\right]-\beta^{-1} S\left(\varrho_{y}\right)\right\} \\
& +\lambda\left\{\operatorname{tr}\left[\left(H+\alpha Q^{2}\right) \varrho_{z}\right]-\beta^{-1} S\left(\varrho_{z}\right)\right\} \\
= & (1-\lambda)\left\{E(y)+\alpha y^{2}\right\}+\lambda\left\{E(z)+\alpha z^{2}\right\} .
\end{aligned}
$$

Lemma 11. The state $\varrho_{x}$ depends continuously on $x \in \mathbb{R}^{3 N}$ in trace norm.

Proof. We prove continuity at $x=0$ only

Since $E(x)+\alpha x^{2}$ is convex

$$
\mathscr{E}\left(\varrho_{x}\right)=E(x)
$$

is continuous and hence bounded as $|x| \rightarrow 0$. Equation (2.3) now implies that $S\left(\varrho_{x} \mid e^{-K}\right)$ is bounded and Lemma 1 implies that

$$
\operatorname{tr}\left[\left(H+\alpha Q^{2}\right) \varrho_{x}\right]
$$

is bounded as $|x| \rightarrow 0$. By Lemma 7 there is a sequence $x(n) \rightarrow 0$ such that $\varrho_{n(n)}$ converges in trace norm, to $\sigma$ say. The lower semi-continuity of $\mathscr{E}$ implies that

$$
\begin{aligned}
\mathscr{E}(\sigma) & \leqq \liminf _{n \rightarrow \infty} \mathscr{E}\left(\varrho_{x(n)}\right) \\
& =\liminf _{n \rightarrow \infty} E(x(n)) \\
& =E(0) .
\end{aligned}
$$


Also Corollary 6 implies that $\operatorname{tr}[Q \sigma]=0$. The uniqueness of the minimum in (3.1) now implies that $\sigma=\varrho_{0}$. Since $\varrho_{0}$ is the only possible limit point of $\varrho_{x}$ as $|x| \rightarrow 0$ we see again by Lemma 7 that $\left\|\varrho_{x}-\varrho_{0}\right\|_{1} \rightarrow 0$ as $|x| \rightarrow 0$.

For the proof of the following lemma, and for the rest of the paper, we make the following strengthened hypothesis on the potential $V$, namely

$$
\left\|V\left(H_{0}+1\right)^{-1 / 2}\right\|<\infty \text {. }
$$

It is a consequence of (1.2) and the uncertainty principle that this condition is satisfied if $V$ arises from two-body Coulomb interactions.

Lemma 12. The function $E(x)$ is differentiable on $\mathbb{R}^{3 N}$.

Proof. We prove differentiability only at $x=0$. If we define

$$
\sigma_{x}=e^{-i P \cdot x} \varrho_{0} e^{i P \cdot x},
$$

where $P_{r}$ are the usual momentum operators then

$$
\operatorname{tr}\left[Q \sigma_{x}\right]=x \text {. }
$$

Hence

$$
\begin{aligned}
E(x) & \leqq \operatorname{tr}\left[H \sigma_{x}\right]-\beta^{-1} S\left(\sigma_{x}\right)+\alpha W\left(\sigma_{x}\right) \\
& =\operatorname{tr}\left[V \sigma_{x}\right]+c,
\end{aligned}
$$

where

$$
c=\operatorname{tr}\left[H_{0} \varrho_{0}\right]-\beta^{-1} S\left(\varrho_{0}\right)+\alpha W\left(\varrho_{0}\right)
$$

by the space translation invariance of $H_{0}, S, W$. We thus get

$$
\begin{aligned}
E(x) & \leqq G(x) \\
& =\operatorname{tr}\left[V \sigma_{x}\right]-\operatorname{tr}\left[V \varrho_{0}\right]+E(0) .
\end{aligned}
$$

Now $\operatorname{tr}\left[P^{2} \varrho_{0}\right]<\infty$ so

$$
\varrho_{0}=\left(1+P^{2}\right)^{-1 / 2} \mu\left(1+P^{2}\right)^{-1 / 2}
$$

for some $\mu \in \mathscr{T}(\mathscr{H})$. Therefore

$$
\begin{aligned}
G(x)-G(0)= & \operatorname{tr}\left[V \sigma_{x}\right]-\operatorname{tr}\left[V \sigma_{0}\right] \\
= & \operatorname{tr}\left[V\left(e^{-i P \cdot x}-1\right) \varrho_{0} e^{i P \cdot x}\right] \\
& -\operatorname{tr}\left[V \varrho_{0}\left(e^{i P \cdot x}-1\right)\right] \\
= & \operatorname{tr}\left[\left(1+P^{2}\right)^{-1 / 2} V \cdot\left(e^{-i P \cdot x}-1\right)\left(1+P^{2}\right)^{-1 / 2} \cdot \mu e^{i P \cdot x}\right] \\
& -\operatorname{tr}\left[V\left(1+P^{2}\right)^{-1 / 2} \cdot \mu \cdot\left(1+P^{2}\right)^{-1 / 2}\left(e^{i P \cdot x}-1\right)\right]
\end{aligned}
$$

from which the differentiability of $G(x)$ at $x=0$ easily follows. Now

$$
\begin{aligned}
E(x)+\alpha x^{2} & \leqq G(x)+\alpha x^{2} \\
& =G(0)+x \cdot G^{\prime}(0)+o(|x|) \\
& =E(0)+x \cdot G^{\prime}(0)+o(|x|)
\end{aligned}
$$


as $|x| \rightarrow 0$. The convexity of the left-hand side implies that

$$
E(x)+\alpha x^{2} \geqq E(0)+x \cdot G^{\prime}(0)
$$

for all $x \in \mathbb{R}^{3 N}$. Hence $E(x)$ is differentiable at $x=0$ with $E^{\prime}(0)=G^{\prime}(0)$.

The relevance of the function $E(x)$ to the metastable states is revealed in the next theorem.

Theorem 13. The state $\varrho \in X$ is a metastable state with respect to $\mathscr{E}$ if and only if $\varrho=\varrho_{x}$ for some $x \in \mathbb{R}^{3 N}$ and $E$ has a local minimum at $x$.

Proof. Let $\varrho$ be a metastable state with $\operatorname{tr}[Q \varrho]=x$, and for $0<\lambda<1$ put

$$
\sigma_{\lambda}=(1-\lambda) \varrho+\lambda \varrho_{x} .
$$

Then $\operatorname{tr}\left[Q \sigma_{\lambda}\right]=x$ and

$$
\begin{aligned}
\mathscr{E}\left(\sigma_{\lambda}\right) & =\mathscr{F}_{x}\left(\sigma_{\lambda}\right) \\
& \leqq(1-\lambda) \mathscr{F}_{x}(\varrho)+\lambda \mathscr{F}_{x}\left(\varrho_{x}\right) \\
& =(1-\lambda) \mathscr{E}(\varrho)+\lambda \mathscr{E}\left(\varrho_{x}\right) \\
& \leqq \mathscr{E}(\varrho)
\end{aligned}
$$

with equality if and only if $\varrho=\varrho_{x}$. Since $\left\|\sigma_{\lambda}-\varrho\right\|_{1} \rightarrow 0$ as $\lambda \rightarrow 0$ the metastability of $\varrho$ implies that $\varrho=\varrho_{x}$. Lemma 11 now implies that $E$ has a local minimum at $x$.

Conversely suppose that $\varrho_{x}$ is not a metastable state. Then there is a sequence $\sigma_{n} \in X$ such that $\left\|\sigma_{n}-\varrho_{x}\right\|_{1} \rightarrow 0$ and $\mathscr{E}\left(\sigma_{n}\right)<\sigma\left(\varrho_{x}\right)$ for all $n$. If $x_{n}=\operatorname{tr}\left[Q \sigma_{n}\right]$ then Corollary 6 implies that $x_{n} \rightarrow x$. Also

$$
E\left(x_{n}\right) \leqq \mathscr{E}\left(\sigma_{n}\right)<\mathscr{E}\left(\varrho_{x}\right)=E(x)
$$

so $E$ does not have a local minimum at $x$. Hence if $E$ does have a local minimum at $x, \varrho_{x}$ must be a metastable state.

\section{Characterization of Metastable States}

The next step in our analysis depends upon the introduction of the function $F$ on $\mathbb{R}^{3 N}$ defined by

$$
F(x)=\min \left\{\mathscr{F}_{x}(\varrho): \varrho \in X\right\} .
$$

For each $x, \mathscr{F}_{x}$ is a strictly convex lower semi-continuous functional on $X$ which takes its minimum at the Gibbs state

$$
\left.\sigma_{x}=e^{-\beta\left\{H+\alpha(Q-x)^{2}\right\}} / \operatorname{tr}\left[e^{-\beta\left\{H+\alpha(Q-x)^{2}\right.}\right\}\right]
$$

from which we obtain

$$
F(x)=-\beta^{-1} \log \operatorname{tr}\left[e^{-\beta\left\{H+\alpha(Q-x)^{2}\right.}\right]
$$


so that $F$ is in principle computable. It is clear from the definition that

$$
E(x) \geqq F(x)
$$

for all $x \in \mathbb{R}^{3 N}$.

Lemma 14. The function $F(x)-\alpha x^{2}$ is concave and differentiable on $\mathbb{R}^{3 N}$. Moreover

$$
F^{\prime}(x)=-2 \alpha \operatorname{tr}\left[(Q-x) \sigma_{x}\right] .
$$

Proof. If $x, y, z \in \mathbb{R}^{3 N}$ and $0<\lambda<1$ and

$$
x=(1-\lambda) y+\lambda z
$$

then

$$
\begin{aligned}
F(x)-\alpha x^{2}= & \operatorname{tr}\left[\left(H+\alpha Q^{2}-2 \alpha Q \cdot x\right) \sigma_{x}\right]-\beta^{-1} S\left(\sigma_{x}\right) \\
= & (1-\lambda)\left\{\operatorname{tr}\left[\left(H+\alpha Q^{2}-2 \alpha Q \cdot y\right) \sigma_{x}\right]-\beta^{-1} S\left(\sigma_{x}\right)\right\} \\
& \left.+\lambda\left\{\operatorname{tr}\left[H+\alpha Q^{2}-2 \alpha Q \cdot z\right) \sigma_{x}\right]-\beta^{-1} S\left(\sigma_{x}\right)\right\} \\
\geqq & (1-\lambda)\left\{F(y)-\alpha y^{2}\right\}+\lambda\left\{F(z)-\alpha z^{2}\right\} .
\end{aligned}
$$

This proves the first statement. From the identity

$$
F(x)-\alpha x^{2}=-\beta^{-1} \log \operatorname{tr}\left[e^{-\beta\left\{H+\alpha Q^{2}-2 \alpha Q \cdot x\right\}}\right]
$$

we see that the other statements follow provided

$$
\begin{aligned}
& \frac{\partial}{\partial x} \operatorname{tr}\left[e^{-\beta\left\{H+\alpha Q^{2}-2 \alpha Q \cdot x\right\}}\right] \\
& \quad=\operatorname{tr}\left[2 \beta \alpha Q e^{-\beta\left\{H+\alpha Q^{2}-2 \alpha Q \cdot x\right\}}\right] .
\end{aligned}
$$

This may be proved by use of the Dyson expansion if $Q$ is replaced by a bounded operator, and the required equality may then be obtained by an approximation procedure.

Note that Corollary 25.5.1 of [10] may be used to establish that $E$ and $F$ are actually continuously differentiable on $\mathbb{R}^{3 N}$.

Theorem 15. The following three conditions are equivalent.

(i) $E(x)=F(x)$,

(ii) $F^{\prime}(x)=0$,

(iii) $E^{\prime}(x)=0$.

Proof. Since $E(x)$ and $F(x)$ are both defined by minimizing $\mathscr{F}_{x}(\varrho)$, but with constraints in the first case, and since in both cases the minimizing state is unique, we see that (i) holds if and only if $\sigma_{x}=\varrho_{x}$, or equivalently if

$$
\operatorname{tr}\left[Q \sigma_{x}\right]=x .
$$

Lemma 14 shows that this is equivalent to (ii). If (i) holds then $E(x)=F(x)$, $F^{\prime}(x)=0, E(y) \geqq F(y)$ for all $y \in \mathbb{R}^{3 N}$ together imply $E^{\prime}(x)=0$.

If $E(x)>F(x)$ we have

$$
\operatorname{tr}\left[Q \varrho_{x}\right]=x, \quad \operatorname{tr}\left[Q \sigma_{x}\right]=y \neq x .
$$


If $0<\lambda<1$ and

$$
z=(1-\lambda) x+\lambda y
$$

and

$$
\mu=(1-\lambda) \varrho_{x}+\lambda \sigma_{x}
$$

then

$$
\operatorname{tr}[Q \mu]=z
$$

and

$$
\begin{aligned}
\mathscr{F}_{z}(\mu)= & \operatorname{tr}\left[\left\{H+\alpha(Q-z)^{2}\right\} \mu\right]-\beta^{-1} S(\mu) \\
= & \operatorname{tr}\left[\left\{H+\alpha(Q-x)^{2}\right\} \mu\right]-\beta^{-1} S(\mu)-\alpha(z-x)^{2} \\
\leqq & (1-\lambda)\left\{\operatorname{tr}\left[\left\{H+\alpha(q-x)^{2}\right\} \varrho_{x}\right]-\beta^{-1} S\left(\varrho_{x}\right)\right\} \\
& +\lambda\left\{\operatorname{tr}\left[\left\{H+\alpha(Q-x)^{2}\right\} \sigma_{x}\right]-\beta^{-1} S\left(\sigma_{x}\right)\right\} \\
& -\alpha \lambda^{2}(y-x)^{2} \\
= & (1-\lambda) E(x)+\lambda F(x)-\alpha \lambda^{2}(y-x)^{2} .
\end{aligned}
$$

Hence

$$
\begin{aligned}
E(z)-E(x) & \leqq \mathscr{F}_{z}(\mu)-E(x) \\
& \leqq-\lambda\{E(x)-F(x)\}-\alpha \lambda^{2}(y-x)^{2}
\end{aligned}
$$

which implies that

$$
E^{\prime}(x) \cdot(y-x) \leqq-\{E(x)-F(x)\}
$$

so $E^{\prime}(x) \neq 0$.

We have thus shown that although $E$ and $F$ are not the same function, they have the same turning points. By combining (3.2) with Lemma 14 we get the further inequality

$$
2 \alpha E^{\prime}(x) \cdot F^{\prime}(x) \geqq E(x)-F(x)
$$

which may be used to obtain more relationships between $E$ and $F$.

Theorem 16. Metastable states are states of the form

$$
\sigma_{x}=e^{-\beta\left\{H+\alpha(Q-x)^{2}\right\}} / \operatorname{tr}\left[e^{-\beta\left\{H+\alpha(Q-x)^{2}\right\}}\right],
$$

where $x \in \mathbb{R}^{3 N}$ is one of the points for which

$$
\operatorname{tr}\left[Q \sigma_{x}\right]=x .
$$

The proof is just a matter of drawing together Theorem 13, Lemma 14, and Theorem 15. For each $0<\alpha, \beta<\infty$ we obtain a family of "modified Gibbs states" $\sigma_{x}$ depending on $x \in \mathbb{R}^{3 N}$. The side condition will then in general restrict $x$ to one of a few discrete values. Each such value $x \in \mathbb{R}^{3 N}$ describes a "classical" configuration of the atoms into a molecule as considered in traditional approaches to the problem of molecular structure. 
Theorem 17. If $\beta^{-1}=0$ and $0<\alpha<\infty$ then the metastable states are of the form

$$
\sigma_{x}=\left|\psi_{x}\right\rangle\left\langle\psi_{x}\right|
$$

where $\psi_{x}$ is the ground state of $\left\{H+\alpha(Q-x)^{2}\right\}$ and $x \in \mathbb{R}^{3 N}$ is one of the points for which

$$
\left\langle Q \psi_{x}, \psi_{x}\right\rangle=x
$$

The proof of this degenerate case is as before if one replaces "Gibbs state" by "ground state" throughout.

\section{Metastable States in a Weak Electric Field}

As an illustrative example, we apply the above ideas to a two-body system at zero temperature in a weak external electric field. After eliminating the centre of mass motion the zero field Hamiltonian $H$ on $\mathscr{H}=L^{2}\left(\mathbb{R}^{3}\right)$ is taken to be

$$
H=-\frac{1}{2 m} \Delta+V \text {, }
$$

where

$$
\left\|V(1-\Delta)^{-1 / 2}\right\|<\infty
$$

and

$$
\lim _{|x| \rightarrow \infty} V(x)=0
$$

To reduce the complexity we also suppose that $V$ is central. We assume that $H$ has a (non-degenerate, central) ground state $\varphi_{0}$ with energy $E_{0}<0$. Then

$$
\operatorname{Sp}(H) \cong\left\{E_{0}\right\} \cup\left[E_{1}, \infty\right),
$$

where $E_{1}=0$ if $\varphi_{0}$ is the only bound state of $H$ and $E_{1}$ is the energy of the first excited state otherwise. We investigate the existence of a metastable state near to $\varphi_{0}$ in the external field $(\gamma A \cdot Q)$, where $A \in \mathbb{R}^{3}$ is a fixed unit vector and $\gamma>0$ is sufficiently small. For a discussion of the relationship between our approach and that of $[3-5]$ see the next section.

Since $\mathscr{E}$ is concave on $X$ when $\beta^{-1}=0$, we may restrict attention throughout to pure states and redefine $\mathscr{E}$ on $\{\psi \in \mathscr{H}:\|\psi\|=1\}$ by

$$
\mathscr{E}(\psi)=\langle H \psi, \psi\rangle-\gamma\langle A \cdot Q \psi, \psi\rangle+\alpha W(\psi),
$$

where

$$
\left.W(\psi)=\sum_{r=1}^{3}\left\langle Q_{r}^{2} \psi, \psi\right\rangle-\left\langle Q_{r} \psi, \psi\right\rangle^{2}\right\} .
$$

The function $E$ on $\mathbb{R}^{3}$ is given by

$$
E(x)=\min \left\{\left\langle H_{x} \psi, \psi\right\rangle:\|\psi\|=1 \quad \text { and } \quad\langle Q \psi, \psi\rangle=x\right\},
$$


where

$$
H_{x}=H-\gamma A \cdot Q+\alpha(Q-x)^{2} .
$$

This operator is self-adjoint and semibounded with pure point spectrum, for all $\alpha>0$. The minimum in (4.1) exists by Theorem 9 . It is clear that $E(x) \geqq F(x)$ for all $x \in \mathbb{R}^{3}$, where $F(x)$ is the ground state energy of $H_{x}$. By Theorem 17 we can obtain metastable states by finding the local minima of $F(x)$, and our goal is to locate these when $\alpha, \gamma>0$ are both very small. Note that if $\alpha=0$ the Hamiltonian $H_{x}$ is not semibounded, so no true ground state exists.

Lemma 18. The minimum of $F(x)$ within the set $\{x:|x| \leqq c\}$ occurs when $x=\lambda A$ for some $0 \leqq \lambda \leqq c$.

Proof. If $L(y)$ is the ground state energy of $\left(H-y \cdot Q+\alpha Q^{2}\right)$ then

$$
F(x)-\alpha x^{2}=L(\gamma A-2 \alpha x) .
$$

Now $L(y)$ is concave and central on $\mathbb{R}^{3}$, so it has its maximum at $y=0$ and is a strictly monotonically decreasing function of $|y|$. If we minimize $F(x)$ with $|x|$ fixed, we see that $x$ must be a positive multiple of $A$. The abolute minimum within $\{x:|x| \leqq c\}$ is then obtained by varying $|x|$.

The following lemma shows that if $\alpha>0$ is too small relative to $\gamma>0$, then there is no metastable state close to $\varphi_{0}$.

Lemma 19. Given $c>0$ and $\gamma_{0}>0$ there exists $\varepsilon>0$ such that $F(x)$ has no local minimum within $\{x:|x|<c\}$ if $0<\gamma<\gamma_{0}$ and $0<\alpha \gamma^{-2}<\varepsilon$.

Proof. If $\|\varphi\|=1$ and $\langle Q \varphi, \varphi\rangle=x$ then

$$
\begin{aligned}
& \langle H \varphi, \varphi\rangle-\gamma\langle A \cdot Q \varphi, \varphi\rangle+\alpha\left\langle(Q-x)^{2} \varphi, \varphi\right\rangle \\
& \quad \geqq E_{0}-\gamma|x| \geqq E_{0}-\gamma_{0} c .
\end{aligned}
$$

Hence

$$
E(x)>E_{0}-\gamma_{0} c .
$$

On the other hand if $c_{0}$ is chosen so that

$$
\varphi(y)=c_{0} \exp \left\{-\left(y-x^{-1 / 2} A\right)^{2}\right\}
$$

is a unit vector then

$$
\begin{aligned}
F(x) & \leqq\langle H \varphi, \varphi\rangle-\gamma\langle A \cdot Q \varphi, \varphi\rangle+\alpha\left\langle(Q-x)^{2} \varphi, \varphi\right\rangle \\
& =\langle H \varphi, \varphi\rangle-\gamma \alpha^{-1 / 2}+\alpha\left\|\alpha^{-1 / 2} A-x\right\|^{2}+\alpha c_{1}^{2} \\
& \leqq\langle H \varphi, \varphi\rangle-\varepsilon^{-1 / 2}+\left(1+\alpha^{1 / 2} c\right)^{2}+\alpha c_{1}^{2} \\
& \leqq\langle H \varphi, \varphi\rangle-\varepsilon^{-1 / 2}+c_{1}^{2}\left(1+\varepsilon^{1 / 2} \gamma_{0} c\right)^{2}+\varepsilon \gamma_{0}^{2} c_{1}^{2} .
\end{aligned}
$$

Since $\langle H \varphi, \varphi\rangle$ has a uniform bound with respect to $\alpha>0$ we see that if $\varepsilon>0$ is small enough then

$$
F(x)<E(x)
$$

so $F$ does not have a local minimum at $x$, by Theorem 15 . 
From now on we put $\alpha=a \gamma^{2}$ where $a>0$ is sufficiently large, but fixed, as $\gamma \rightarrow 0$. We use the formal perturbation expansion

$$
\begin{aligned}
& \left\{H-\gamma A \cdot Q+a \gamma^{2}(Q-x)^{2}\right\}\left\{\varphi_{0}+\gamma \psi_{1}+\gamma^{2} \psi_{2}+\ldots\right\} \\
& \quad=\left\{E_{0}+\gamma F_{1}+\gamma^{2} F_{2}+\ldots\right\}\left\{\varphi_{0}+\gamma \psi_{1}+\gamma^{2} \psi_{2}+\ldots\right\}
\end{aligned}
$$

as a guide for determining approximations to the ground state energy $F(x)$. Note that the spherical symmetry of $\varphi_{0}$ implies that

$$
\left\langle A \cdot Q \varphi_{0}, \varphi_{0}\right\rangle=0
$$

so $F_{1}=0$. Moreover

$$
\psi_{1}=S(A \cdot Q) \varphi_{0},
$$

where the bounded operators $S, X$ are defined by

$$
S=\left(H-E_{0}\right)^{-1} X
$$

and

$$
X=1-\left|\varphi_{0}\right\rangle\left\langle\varphi_{0}\right| .
$$

We shall need the following technical lemma.

Lemma 20. One has

$$
\left\|Q_{s} Q_{r} \psi_{1}\right\|<\infty
$$

for all $r, s$.

Proof. We refer to [12] or [9, Theorem 13.70] for the proof that

$$
\left\||Q|^{n} \varphi_{0}\right\|<\infty
$$

for all $n \geqq 0$, and start by proving that

$$
\left\|Q_{r} \psi_{1}\right\|<\infty \text {. }
$$

Since

$$
Q_{r} \psi_{1}=X Q_{r} \psi_{1}+\left\langle S(Q \cdot A) \varphi_{0}, Q_{r} \varphi_{0}\right\rangle \varphi_{0}
$$

it is sufficient to prove the finiteness of the norm of

$$
\begin{aligned}
X Q_{r} \psi_{1}= & X Q_{r} S(Q \cdot A) \varphi_{0} \\
= & S\left(H-E_{0}\right) Q_{r} S(Q \cdot A) \varphi_{0} \\
= & S Q_{r}\left(H-E_{0}\right) S(Q \cdot A) \varphi_{0} \\
& +S\left[\left(H-E_{0}\right), Q_{r}\right] S(Q \cdot A) \varphi_{0} \\
= & S Q_{r}(Q \cdot A) \varphi_{0}-\frac{i}{m} S P_{r} S(Q \cdot A) \varphi_{0} .
\end{aligned}
$$


One sees that

$$
\begin{aligned}
\left\|X Q_{r} \psi_{1}\right\| & \leqq\|S\|\left\|Q_{s}(Q \cdot A) \varphi_{0}\right\|+\frac{1}{m}\left\|S P_{r} S\right\|\left\|(Q \cdot A) \varphi_{0}\right\| \\
& <\infty .
\end{aligned}
$$

Similarly

$$
Q_{s} Q_{r} \psi_{1}=X Q_{s} Q_{r} \psi_{1}+\left\langle S(Q \cdot A) \varphi_{0}, Q_{r} Q_{s} \varphi_{0}\right\rangle \varphi_{0}
$$

so (4.3) follows once one proves the finiteness of the norm of

$$
\begin{aligned}
X Q_{s} Q_{r} \psi_{1}= & X Q_{s} Q_{r} S(Q \cdot A) \varphi_{0} \\
= & S\left(H-E_{0}\right) Q_{s} Q_{r} S(Q \cdot A) \varphi_{0} \\
= & S Q_{s} Q_{r}\left(H-E_{0}\right) S(Q \cdot A) \varphi_{0} \\
& +S\left[\left(H-E_{0}\right), Q_{s} Q_{r}\right] S(Q \cdot A) \varphi_{0} \\
= & S Q_{s} Q_{r}(Q \cdot A) \varphi_{0} \\
& +\frac{1}{2 m} S\left[P^{2}, Q_{s} Q_{r}\right] S(Q \cdot A) \varphi_{0} .
\end{aligned}
$$

The first term has finite norm by (4.4) and the second term equals

$$
\begin{aligned}
& -i m^{-1} S\left(P_{s} Q_{r}+Q_{s} P_{r}\right) S(Q \cdot A) \varphi_{0} \\
& =-i m^{-1} S\left(i \delta_{r s}+P_{r} Q_{s}+P_{s} Q_{r}\right) S(Q \cdot A) \varphi_{0} \\
& =-m^{-1} \delta_{r s} S \psi_{1}-i m^{-1}\left(S P_{r}\right)\left(Q_{s} \psi_{1}\right)-i m^{-1}\left(S P_{s}\right)\left(Q_{r} \psi_{1}\right)
\end{aligned}
$$

which has finite norm by (4.5).

Lemma 21. The inequality

$$
\begin{aligned}
F(x) \leqq & E_{0}-\gamma^{2}\left\langle(Q \cdot A) S(Q \cdot A) \varphi_{0}, \varphi_{0}\right\rangle \\
& +a \gamma^{2}\left\langle Q^{2} \varphi_{0}, \varphi_{0}\right\rangle+a \gamma^{2} x^{2}+O\left(\gamma^{3}\right)
\end{aligned}
$$

holds uniformly for $|x|<c$ as $\gamma \downarrow 0$.

Proof. If $\varphi=\varphi_{0}+\gamma \psi_{1}$ then

$$
\begin{aligned}
\left\langle H_{x} \varphi, \varphi\right\rangle= & \left\langle H\left(\varphi_{0}+\gamma \psi_{1}\right), \varphi_{0}+\gamma \psi_{1}\right\rangle \\
& -\gamma\left\langle A \cdot Q\left(\varphi_{0}+\gamma \psi_{1}\right), \varphi_{0}+\gamma \psi_{1}\right\rangle \\
& +a \gamma^{2}\left\langle(Q-x)^{2}\left(\varphi_{0}+\gamma \psi_{1}\right), \varphi_{0}+\gamma \psi_{1}\right\rangle \\
= & E_{0}+\gamma^{2}\left\langle H \psi_{1}, \psi_{1}\right\rangle \\
& -\gamma^{2}\left\langle A \cdot Q \varphi_{0}, \psi_{1}\right\rangle-\gamma^{2}\left\langle\psi_{1}, A \cdot Q \varphi_{0}\right\rangle \\
& +a \gamma^{2}\left\langle(Q-x)^{2} \varphi_{0}, \varphi_{0}\right\rangle+O\left(\gamma^{3}\right) \\
= & E_{0}-\gamma^{2}\left\langle(Q \cdot A) S(Q \cdot A) \varphi_{0}, \varphi_{0}\right\rangle \\
& +E_{0} \gamma^{2}\left\langle\psi_{1}, \psi_{1}\right\rangle+a \gamma^{2}\left\langle(Q-x)^{2} \varphi_{0}, \varphi_{0}\right\rangle+O\left(\gamma^{3}\right) .
\end{aligned}
$$


Since

$$
\begin{aligned}
\|\varphi\|^{-2} & =\left(\left\|\varphi_{0}\right\|^{2}+\gamma^{2}\left\|\psi_{1}\right\|^{2}\right)^{-1} \\
& =1-\gamma^{2}\left\|\psi_{1}\right\|^{2}+O\left(\gamma^{4}\right)
\end{aligned}
$$

we conclude that

$$
\begin{aligned}
F(x) \leqq & \|\varphi\|^{-2}\left\langle H_{x} \varphi, \varphi\right\rangle \\
= & E_{0}-E_{0} \gamma^{2}\left\|\psi_{1}\right\|^{2}+\gamma^{2}\left\langle H \psi_{1}, \psi_{1}\right\rangle \\
& -\gamma^{2}\left\langle A \cdot Q \varphi_{0}, \psi_{1}\right\rangle-\gamma^{2}\left\langle\psi_{1}, A \cdot Q \varphi_{0}\right\rangle \\
& +a \gamma^{2}\left\langle Q^{2} \varphi_{0}, \varphi_{0}\right\rangle+a \gamma^{2} x^{2}+O\left(\gamma^{3}\right)
\end{aligned}
$$

which yields the stated inequality upon simplification.

We next obtain a crude lower bound to the energy $G(x)$ of the first excited state of $H_{x}$. The condition (4.8) below states that states that $\gamma>0$ should be small and $a>0$ should be large. We shall henceforth always assume that this condition is satisfied.

Lemma 22. If $x \in \mathbb{R}^{3}$ then

$$
G(x) \geqq E_{1}-\gamma A \cdot x-\frac{1}{4 a} .
$$

In particular if $|x| \leqq c$ and $\gamma$, a satisfy

$$
\gamma c+\frac{1}{4 a} \leqq \frac{1}{2}\left(E_{1}-E_{0}\right)
$$

then

$$
G(x) \geqq \frac{1}{2}\left(E_{0}+E_{1}\right) .
$$

Proof. The quadratic form inequality

$$
\begin{aligned}
H_{x}= & H-\gamma Q \cdot A+a \gamma^{2}(Q-x)^{2} \\
= & H+a \gamma^{2} Q^{2}-\left(\gamma A+2 a \gamma^{2} x\right) \cdot Q+a \gamma^{2} x^{2} \\
= & H+a \gamma^{2}\left(Q-x-\frac{A}{2 a \gamma}\right)^{2} \\
& -a \gamma^{2}\left(x+\frac{A}{2 a \gamma}\right)^{2}+a \gamma^{2} x^{2} \\
\geqq & H-\gamma A \cdot x-\frac{1}{4 a}
\end{aligned}
$$

leads to (4.7) by a standard result. The proof of (4.9) is now elementary

Theorem 23. If $a>0$ is large enough then

$$
\begin{aligned}
F(x)= & E_{0}-\gamma^{2}\left\langle(Q \cdot A) S(Q \cdot A) \varphi_{0}, \varphi_{0}\right\rangle \\
& +a \gamma^{2}\left\langle Q^{2} \varphi_{0}, \varphi_{0}\right\rangle+a \gamma^{2} x^{2}+O\left(\gamma^{3}\right)
\end{aligned}
$$

uniformly for $|x| \leqq c$ as $\gamma \downarrow 0$. 
Proof. By Lemma 21 we need only prove a similar lower bound for $F(x)$, and we do this by using Temple's inequality $\left[9\right.$, p. 84]. If $\varphi=\varphi_{0}+\gamma \psi_{1}$, as before, we have

$$
F(x) \geqq\left\langle H_{x} \varphi, \varphi\right\rangle\|\varphi\|^{-2}-\frac{\left\langle H_{x}^{2} \varphi, \varphi\right\rangle\|\varphi\|^{-2}-\left\langle H_{x} \varphi, \varphi\right\rangle^{2}\|\varphi\|^{-4}}{\left\langle H_{x} \varphi, \varphi\right\rangle\|\varphi\|^{-2}-G(x)} .
$$

By Lemmas 21 and 22 the denominator is bounded away from zero as $\gamma \downarrow 0$ provided $a>0$ is large enough. Since also $\|\varphi\| \rightarrow 1$ as $\gamma \downarrow 0$ we have only to show that

$$
\left\langle H_{x}^{2} \varphi, \varphi\right\rangle\|\varphi\|^{2}-\left\langle H_{x} \varphi, \varphi\right\rangle^{2}=O\left(\gamma^{3}\right) .
$$

Now application of Lemma 20 shows that

$$
\begin{aligned}
H_{x} \varphi= & \left\{H-\gamma(A \cdot Q)+a \gamma^{2}(Q-x)^{2}\right\}\left(\varphi_{0}+\psi_{1}\right) \\
= & E_{0} \varphi_{0}=+\gamma\left\{H \psi_{1}-(A \cdot Q) \varphi_{0}\right\} \\
& +\gamma^{2}\left\{a(Q-x)^{2} \varphi_{0}-(A \cdot Q) \psi_{1}\right\}+O\left(\gamma^{3}\right) \\
= & E_{0} \varphi_{0}+\gamma E_{0} \psi_{1}+\gamma^{2}\left\{a(Q-x)^{2} \varphi_{0}-(A \cdot Q) \psi_{1}\right\}+O\left(\gamma^{3}\right) .
\end{aligned}
$$

Therefore

$$
\begin{aligned}
\left\langle H_{x}^{2}\right. & \varphi, \varphi\rangle\|\varphi\|^{2} \\
= & \|\varphi\|^{2}\left\{E_{0}^{2}+2 E_{0} \gamma^{2} a\left\langle(Q-x)^{2} \varphi_{0}, \varphi_{0}\right\rangle\right. \\
& -E_{0} \gamma^{2}\left\langle\varphi_{0},(A \cdot Q) \psi_{1}\right\rangle-E_{0} \gamma^{2}\left\langle(A \cdot Q) \psi_{1}, \varphi_{0}\right\rangle \\
& \left.+\gamma^{2} E_{0}^{2}\left\langle\psi_{1}, \psi_{1}\right\rangle+O\left(\gamma^{3}\right)\right\} \\
= & E_{0}^{2}+2 E_{0} \gamma^{2} a\left\langle(Q-x)^{2} \varphi_{0}, \varphi_{0}\right\rangle \\
& -2 E_{0} \gamma^{2}\left\langle(A \cdot Q) S(A \cdot Q) \varphi_{0}, \varphi_{0}\right\rangle \\
& +2 \gamma^{2} E_{0}^{2}\left\langle\psi_{1}, \psi_{1}\right\rangle+O\left(\gamma^{3}\right) .
\end{aligned}
$$

We finally obtain (4.10) by comparing the above equation with (4.6).

Corollary 24. If $a>0$ is sufficiently large and $\gamma>0$ is sufficiently small then the function $F(x)$ has a minimum in $\{x:|x|<c\}$ and that minimum occurs for $x=\lambda A$ where

$$
0 \leqq \lambda=O\left(\gamma^{1 / 2}\right)
$$

Moreover

$$
\min _{|x| \leqq c} F(x)=E_{0}+O\left(\gamma^{2}\right) .
$$

\section{Interpretation of the Results}

We have described a method for defining a metastable state of an atom at zero temperature in a non-zero electric field. An entirely different way of approaching this problem described in [3-5] is to study the spectrum of the Hamiltonian $H-\gamma(A \cdot Q)$ after a transformation known as complex scaling. It turns out that this 
operator then has a complex eigenvalue $K(\gamma)$ with an asymptotic expansion

$$
K(\gamma)=E_{0}+K_{1} \gamma+K_{2} \gamma^{2}+\ldots,
$$

where the (real) coefficients $K_{r}$ are obtained from the formal perturbation expansion

$$
\begin{aligned}
& \{H-\gamma(A \cdot Q)\}\left\{\varphi_{0}+\gamma \eta_{1}+\gamma^{2} \eta_{2}+\ldots\right\} \\
& \quad=\left\{E_{0}+K_{1} \gamma+K_{2} \gamma^{2}+\ldots\right\}\left\{\varphi_{0}+\gamma \eta_{1}+\gamma^{2} \eta_{2}+\ldots\right\} .
\end{aligned}
$$

While this approach is more intrinsic to the problem, in that no "thermodynamic parameters" $\alpha$ or $a$ appear, it is not very clear what its operational interpretation should be, because the Hamiltonian after complex scaling is no longer self-adjoint. While this may not be regarded as a serious problem, it seems that no prescription in these terms has yet been given for defining a positive temperature metastable state in a non-zero field.

In this particular problem a "better" method than the one we described is to put the atom in the electric field and also in a spherical box of carefully chosen radius, depending on $\gamma$. One should then obtain a real ground state energy which has an asymptotic expansion in $\gamma$ agreeing to all orders with that obtained by the complex scaling method. The advantage of our method is that the constraint introduced by the non-linear term $\alpha W(\varrho)$ is not dependent upon knowing the answer one is looking for, so that our method is a more fundamental one.

Returning to the example of Sect. 4, we give some justification for calling the state determined a metastable state.

Theorem 25. Let $\psi$ be the normalised ground state of the Hamiltonian

$$
H_{x}=H-\gamma A \cdot Q+a \gamma^{2}(Q-x)^{2},
$$

where $x$ is the point determined in Corollary 24 at which $F$ has a local minimum near to zero. Then

$$
(H-\gamma A \cdot Q) \psi=E_{0} \psi+O\left(\gamma^{2}\right)
$$

as $\gamma \downarrow 0$, so that there exists a constant $b<\infty$ such that

$$
\left\|e^{-i(H-\gamma A \cdot Q) t} \psi-e^{-i E_{0} t} \psi\right\| \leqq b \gamma^{2} t
$$

for all $t \geqq 0$.

\section{Proof. Since}

$$
H \psi=F(x) \psi
$$

we have

$$
\begin{aligned}
& \left\|\left(H_{0}-\gamma A \cdot Q\right) \psi-E_{0} \psi\right\| \\
& \quad=\left\|\left(F(x)-E_{0}\right) \psi-a \gamma^{2}(Q-x)^{2} \psi\right\| \\
& \quad \leqq\left|F(x)-E_{0}\right|+a \gamma^{2}\left\|(Q-x)^{2} \psi\right\| \\
& \quad \leqq\left|F(x)-E_{0}\right|+a \gamma^{2} c^{2}+2 a \gamma^{2} c\|Q \psi\|+a \gamma^{2}\left\|Q^{2} \psi\right\| .
\end{aligned}
$$


The proof of (5.1) is completed by showing that $\left\|Q^{2} \psi\right\|$ is uniformly bounded as $\gamma \downarrow 0$. An examination of the proof of [12, Theorem 1] or [9, Theorem 13.70] shows that the various constants involved can be chosen uniformly with respect to small $\gamma>0$ and all $x$ with $|x| \leqq c$. The proof of (5.2) from (5.1) is elementary.

\section{References}

1. Capocaccia, D., Cassandro, M., Olivieri, E.: A study of metastability in the Ising model. Commun. Math. Phys. 39, 185-205 (1974)

2. Davies, E.B. : Symmetry breaking for a non-linear Schrödinger equation. Commun. Math. Phys. 64, 191-210 (1979)

3. Herbst, I.: Dilation analyticity in constant electric field. I. The two body problem. Commun. Math. Phys. 64, 279-298 (1979)

4. Herbst, I., Simon, B.: The Stark effect revisited. Phys. Rev. Lett. 41, 67-69 (1978)

5. Hunziker, W.: Schrödinger operators with electric or magnetic fields. Proc. Int. Conf. Math. Phys., Lausanne, 1979 (to appear)

6. Lavine, R.: Spectral density and sojourn times. Proc. London Ontario Conf. on Atomic Scattering, 1979 (to appear)

7. Lindblad, G.: Expectations and entropy inequalities for finite quantum systems. Commun. Math. Phys. 39, 111-119 (1974)

8. Penrose, O., Lebowitz, J.L.: Towards a rigorous molecular theory of metastability. In: Fluctuation phenomena, studies in statistical mechanics (eds. J.L. Lebowitz, E.W. Montroll). Amsterdam: North-Holland 1979

9. Reed, M., Simon, B.: Methods of modern mathematical physics, Vol. 4. Analysis of operators. London, New York: Academic Press 1978

10. Rockafellar, R.T.: Convex analysis. Princeton, NJ : Princeton University Press 1970

11. Sewell, G.L.: Stability, equilibrium, and metastability in statistical mechanics. Phys. Rep. 57, 307-342 (1980)

12. Simon, B.: Pointwise bounds on eigenfunctions and wave packets in $N$-body quantum systems. II. Proc. Am. Math. Soc. 45, 454-456 (1974)

13. Wehrl, A.: General properties of entropy. Rev. Mod. Phys. 50, 221-260 (1978)

Communicated by J. Ginibre

Received February 11, 1980 
